\section{Motivos de evasão na pós-graduação no Brasil: um instrumento de medida}

\author{
Rodolfo Augusto Matteo Ambiel \\ Ariela Raissa Lima Costa \\ Ana Deyvis Santos Araújo Jesuíno \\ Camila Cardoso Camilo \\ Samanta Romanin Zuchetto
}

\section{RESUMO}

A quantidade de pessoas que iniciam e que concluem as pós-graduações é discrepante, mesmo considerando-se a duração do curso. 0 objetivo deste trabalho foi adaptar a Escala de Motivos de Evasão do Ensino Superior (M-ES) para a Pós-Graduação. Foram realizados dois estudos para tal finalidade. No primeiro, os itens da M-ES foram avaliados por juízes que indicaram a necessidade de criação de novos itens. No segundo, participaram 639 pessoas entre 21 e 62 anos, a maioria da pós-graduação (75\%). Dos modelos testados, a versão de 7 fatores, denominados motivos interpessoais, relacionados à carreira, falta de suporte, desempenho acadêmico, produção científica, reconhecimento acadêmico e institucionais, foi a que apresentou melhor ajuste. Os valores de alfa dos fatores variaram de 0,61 a 0,97 . Sabendo da importância da ciência para o desenvolvimento de qualquer nação, fez-se necessário o desenvolvimento deste estudo, a fim de garantir que os pesquisadores tenham condições adequadas para a conclusão dos cursos e para contribuição com a ciência e sociedade de uma forma geral.

Palavras-chave: evasão; pós-graduação; pesquisa.

\section{ABSTRACT}

\section{Drop-out reasons of graduation in Brazil: a measurement tool}

The number of people starting and completing graduation is discrepant, even considering course duration. The aim was to adapt the Escala de Motivos de Evasão do Ensino Superior (M-ES) (Scale of drop-out reasons in higher education) to the graduation context. Two studies were carried out; in the first, the M-ES items were evaluated by judges, and new ones were constructed according to the new setting. In the second study, 639 people participated, aged between 21 and 62 years, most attending a graduation course $(75 \%)$. The 7 -factor version fitted better, being composed of interpersonal motives, related to career, lack of support, academic performance, scientific production, academic recognition and institutional reasons. Alpha values ranged from 0.61 to 0.97 . Science is fundamental to the development of any nation and it is necessary to ensure that those involved in research might have appropriate conditions to conclude their courses in order to contribute to the desired development.

Keywords: school drop-out; graduation; research.

\section{Sobre os Autores}

R.A.M.A

ORCID: http://orcid.org/0000-

0002-3921-8547

Universidade São Francisco

ambielram@gmail.com

A.R.L.C

ORCID: https://orcid.org/00000002-5942-6466

Universidade São Francisco

arielalima10@gmail.com

A.D.S.A.J

ORCID: http://orcid.org/00000002-7031-7682

Universidade São Francisco

(UFJF) - Juiz de Fora, MG

anadeyvis@gmail.com

C.C.C

ORCID: http://orcid.org/00000002-8619-4904

Universidade São Francisco

camilaccamilo1@gmail.com

S.R.Z

ORCID: http://orcid.org/00000002-4709-5276

Universidade São Francisco

samantazuchetto@hotmail.com

\section{Direitos Autorais}

Este é um artigo aberto e pode ser reproduzido livremente, distribuído, transmitido ou modificado, por qualquer pessoa desde que usado sem fins comerciais. 0 trabalho é disponibilizado sob a licença Creative Commons CC-BY-NC 
A produção científica brasileira tem avançado notoriamente nos últimos cinco anos, com uma taxa de crescimento de $10,7 \%$ ao ano, o que é considerado cinco vezes maior do que a média mundial (Almeida \& Guimarães, 2013). Esse fato está diretamente relacionado com o aumento da quantidade de programas de pósgraduação a nível de mestrado, mestrado profissional e doutorado no país (Almeida \& Guimarães, 2013). 0 número de programas desse tipo no Brasil passou de 1237, em 1998, para 4177, em 2016 (GEOCAPES, 2018), ou seja, mais do que dobrou em um período de dezoito anos. Em 2014, o número de alunos matriculados nos cursos de mestrado foi de 114.341, enquanto o número de titulados em 2016 foi de 49.002. Considerando que um curso de mestrado tem uma duração de até dois anos para ser concluído, esperava-se um valor aproximado entre os matriculados de 2014 e os titulados em 2016, mas somente $42,86 \%$ se titularam. Esses dados geram um questionamento: quais motivos levam os estudantes dos Programas de Pós-Graduação (PPGs) a não concluírem sua formação?

A evasão de discentes de níveis avançados de ensino é um ato voluntário decorrente, principalmente, de um desempenho acadêmico insatisfatório e da não integração social ao novo ambiente, especificamente no primeiro ano do curso (Tinto, 1975; 2007). Assim, o abandono da pós-graduação ocorre por questões pessoais e acadêmicas (Castelló et al., 2017; Demetriou \& Schmitz-Sciborski, 2011; Leijen et al., 2015). Os principais motivos pessoais são despreparo do próprio discente, falta de interesse por pesquisa, questões emocionais e financeiras, relações sociais insatisfatórias com colegas, professores e funcionários, a ausência de programas que contribuam para o enriquecimento curricular, a necessidade de trabalhar ou a dependência de alguém para custear os estudos e a distância de casa, entre outros. Por sua vez, as razões acadêmicas destacadas foram a falta de relação com seus pares, insuficiência ou ausência de supervisão, pressão por produtividade, prazos curtos para realizar a pesquisa e pouca interação com outros pesquisadores (Gardner, 2009; Leijen et al., 2015).

Em uma pesquisa com estudantes espanhóis de doutorado que já pensaram em abandonar o curso, Castelló et al. (2017) discriminaram seis principais razões, que são: (1) não conseguir balancear a vida pessoal e acadêmica, (2) pouca socialização e integração com comunidade científica que envolve problemas de relação com o orientado, (3) falta de motivação por não perceberem relevância social de seus estudos,
(4) escassez de recursos financeiros e tempo para desenvolver a pesquisa, (5) autopercepção negativa acerca das qualificações necessárias para ser um pesquisador e (6) dificuldades emocionais causadas pelas demandas do doutorado. Resultados similares foram encontrados em pesquisas com estudantes finlandeses (Stubb et al., 2011)the study examines how experiences were related to study engagement and to selfreported stress, exhaustion, and anxiety. Altogether 669 doctoral students from the University of Helsinki, Finland, responded a survey. The answers to an open-ended question were content analysed and then statistically compared to well-being and study engagement items. The results showed that there was variation in students' experiences of the scholarly community regarding sociopsychological well-being. More than half of the answers, where students had explicitly described their experience (n $=383$, franceses (van der Haert et al., 2014) e estonianos (Leijen et al., 2015). Esses dados demonstram que dificuldades pessoais e acadêmicas relacionadas a pósgraduação são questões que afetam diversos estudantes, independente da cultura.

Levecque et al. (2017) compararam estudantes belgas de pós-graduação com pessoas da população geral com escolaridade elevada e estudantes de ensino superior. Os autores verificaram que os estudantes de pós-graduação eram mais vulneráveis a experienciar problemas psicológicos e de ter, ou desenvolver, transtornos psiquiátricos devido a preocupações com o curso e dificuldade de participar de interações sociais não relacionadas a universidade. Isso enfatiza a necessidade de ter um olhar cuidadoso sobre as relações desenvolvidas em programas de pós-graduação.

No Brasil, não foi encontrado o mesmo interesse pela temática. Os autores deste manuscrito realizaram uma busca rápida de artigos científicos em maio de 2018, nas bases de dados brasileiras Scielo e PePSIC, utilizando a palavra-chave "evasão", na qual não foi delimitada a data do artigo. Na busca inicial, foram encontrados um total de 228 artigos, sendo 186 do Scielo e 42 do PePSIC. Os títulos, resumos e palavras-chaves de todos os artigos foram lidos e nenhum deles tratavam de evasão de estudantes de pós-graduação à nível de mestrado, doutorado e mestrado profissional. Os poucos estudos encontrados nessa busca, que levantavam uma preocupação com essa população, focaram-se em verificar o nível de estresse de discentes de pós-graduação (Duque et al., 2005; Faro, 2013; Ferreira et al., 2016; Malagris et al., 2009; Paulino et al., 2010).

Apesar da ausência de publicações científicas sobre a 
temática no Brasil, a evasão no ensino superior é explorada há anos em consequência do aumento considerável de ingressantes nas Instituições de Ensino Superior (IES). Esse fato ocorreu em virtude do grande índice de alunos que abandonam seus cursos, o que gerou a necessidade de buscar informações sobre os motivos que levam os alunos a evadirem das IES. Na tentativa de contribuir com a prevenção e para o acompanhamento do fenômeno da evasão no país, Ambiel (2015), construiu a Escala de Motivos para Evasão do Ensino Superior (M-ES).

A M-ES é composta por sete fatores que mensuram motivos institucionais, pessoais, falta de suporte, carreira, desempenho acadêmico, motivos interpessoais e relacionados à autonomia. Identificar quais os motivos para a evasão na pós-graduação contribuiria para a compreensão do fenômeno e elaboração de intervenções para o contexto. Em virtude disso, a presente pesquisa tem por objetivo realizar a adaptação da Escala de Motivos para Evasão do Ensino Superior (Ambiel, 2017) para a pós-graduação.

\section{MÉTODO}

\section{PROCEDIMENTOS DE ADAPTAÇÃO}

A adaptação da Escala de Motivos de Evasão do Ensino Superior (M-ES; Ambiel, 2017) foi realizada em quatro etapas. Primeiro, os 53 itens que compõem a escala foram reescritos para considerar o contexto de pós-graduação, por exemplo, o item "Indecisão sobre continuar ou não meu curso superior atual" foi reescrito para "Indecisão sobre continuar ou não meu curso de pós-graduação atual". Essa modificação foi realizada em 10 itens que evidenciavam o contexto de graduação. Além disso, três pessoas que cursavam e três que abandonaram a pós-graduação foram questionadas acerca dos motivos que as levariam ou levaram a desistir do curso. Mediante essa informação e consulta a artigos científicos que investigaram o tema, foram acrescentados sete itens relativos à relação com o orientador e ao suporte financeiro.

$\mathrm{Na}$ segunda etapa, os itens foram enviados para três juízes doutores em psicologia, com mais de 10 anos de experiência reconhecida em pesquisa na área de psicologia educacional. Eles foram solicitados a avaliar a pertinência do conteúdo do item para o contexto de pósgraduação, bem como a clareza de linguagem dos itens. Um espaço para sugestões foi acrescentado após cada item e após o conjunto dos itens. Segundo as sugestões,
18 itens foram reescritos para apresentar uma linguagem mais clara e coerente com o contexto de pós-graduação. Ainda com base nas sugestões dos juízes, foi retirado um item relativo a motivo relacionado a desempenho acadêmico e foram acrescentados sete itens relativos à dificuldade relacionada a escrita científica e tais itens foram reencaminhados para os juízes analisarem, sendo todos considerados pertinentes e com linguagem clara. Na terceira etapa, o conjunto de 66 itens finais foi divulgado para estudantes de pós-graduação para que eles respondessem. A quarta etapa foi de análise estatística dos dados com a versão de 66 itens.

\section{PARTICIPANTES}

A amostra foi composta por 639 pessoas, com idades entre 21 e 62 anos $(M=29,61 ; D P=5,93)$, sendo $73,6 \%$ mulheres e $66,2 \%$ solteiros. Do total de participantes, $75 \%$ estavam cursando alguma pós-graduação, 21,3\% eram concluintes e 3,4\% eram alunos evadidos. Entre essas pessoas, $76,1 \%$ possuíam bolsa e $27,4 \%$ se dedicavam entre 20 e 30 horas por semana em atividades relacionadas à pós-graduação. A maioria deles (55,9\%) eram alunos de instituições públicas federais, $31,9 \%$ de instituições públicas estaduais, $10,6 \%$ de instituições particulares e $1,6 \%$ de instituições comunitárias. As áreas de investigação mais predominantes foram ciências humanas (18\%), ciências biológicas (17,8\%) e ciências da saúde $(17,1 \%)$. A maioria pertencente à Região Sudeste (56,92\%), seguido da Região Sul $(21,92 \%)$ e das Regiões Nordeste $(9,12 \%)$, Centro-oeste $(6,88)$ e Norte $(5,12 \%)$ em menor quantidade. Em relação à etnia, $70,1 \%$ se autodeclararam brancos, $19,4 \%$ pardos e $3,8 \%$ negros.t.

\section{INSTRUMENTOS}

Escala de Motivos de Evasão do Ensino Superior (M-ES; Ambiel, 2017). Escala composta por 53 itens, respondidos em uma escala de resposta tipo Likert de 5 pontos, sendo 1 = "muito fraco" a 5 = "muito forte", e divididos em sete fatores (Motivo relacionado à Carreira, Motivo Interpessoal, Motivo institucional, Motivo relacionado ao Desempenho Acadêmico, Motivo relacionado à Falta de Suporte, Motivo relacionado à Autonomia e Motivo vocacional). Devido a direitos autorais, os itens originais e da versão adaptada não podem ser divulgados. Apesar disso, vale lembrar que os itens usados como exemplos foram aprovados e autorizados pelo autor do instrumento. 


\section{PROCEDIMENTOS}

O projeto foi aprovado por um comitê de ética em pesquisa (CEP) sob CAAE 76726517.9.0000.5514. A coleta foi iniciada após aprovação do CEP e ocorreu de forma online, com questionário construído na plataforma Google Forms. O link foi divulgado em grupos do Facebook de estudantes de pós-graduação, em que primeiro as pessoas tinham que assinalar concordância com $o$ Termo de Consentimento Livre e Esclarecido (TCLE) para participarem da pesquisa. Os participantes responderam ao questionário sociodemográfico e à versão adaptada da Escala de Motivos de Evasão no Ensino Superior. 0 tempo médio de aplicação foi de 20 minutos.

\section{ANÁLISE DE DADOS}

Os dados da aplicação em estudantes de pósgraduação foram avaliados quanto a estrutura interna, por meio de análise fatorial exploratória. No software $R$ Studio foram utilizados os métodos de extração de Análise Paralela, Bayesian Information Criterion (BIC) e Minimum Avarage Partial (MAP), com rotação oblíqua Oblimin por se compreender que os fatores tem alguma relação (Damásio, 2012). As cargas fatoriais dos itens nos fatores sugeridos foram estimadas no software Mplus. 0 estimador foi o Weighted Least Squares Estimation (WLSMV), com matriz de correlações policóricas, por serem mais adequados para dados ordinais (Asún et al., 2016)maximum likelihood and weighted least squares estimations using Pearson correlation matrices among items are compared. For IFA, diagonally weighted least squares and unweighted least squares estimations using items polychoric correlation matrices are compared. Two hundred and ten conditions were simulated in a Monte Carlo study considering: one to three factor structures (either, independent and correlated in two levels. 0 ajuste do modelo foi verificado por meio dos índices Quiquadrado $\left(\chi^{2}\right)$, Comparative Fit Index (CFI), Tucker-Lewis Index (TLI) e Root Mean Square Error of Approximation (RMSEA). Os valores que indicam um ajuste aceitável são $\chi^{2}$ com menor valor possível, CFI igual ou maior a 0,90 , igual ou maior a 0,95 , e RMSEA igual ou menor a 0,06 (Lai \& Green, 2016). A consistência interna dos fatores foi avaliada por meio do coeficiente alpha de Cronbach.
Os itens ainda foram avaliados pelo pressupostos do modelo Rasch (Bond \& Fox, 2015). Para isso foi utilizado o software Winsteps para verificar os parâmetros de dificuldade dos itens e das pessoas e o ajuste dos itens por meio dos valores de Infit e Outfit. Para Linacre (2014), valores aceitáveis devem estar no intervalo entre 0,5 e 1,5. O Infit indica discrepância nas respostas aos itens, enquanto que o Outfit indica discrepância entre a dificuldade das pessoas e dos itens (Bond \& Fox, 2015). Além disso, foi verificado se existia um funcionamento diferencial dos itens entre bolsistas e não bolsistas, isso é, se existiam itens que eram mais facilmente endossados por um grupo específico (Bond \& Fox, 2015).

\section{RESULTADOS}

\section{Estrutura interna}

Para análise da estrutura interna do instrumento, inicialmente, foi verificado que os itens eram fatoráveis, com índice de Kaiser-Meyer-Olkin (KMO) $=0,96$ ( $p<$ $0,001)$. O segundo passo foi verificar quantos fatores poderiam ser extraídos dos dados. Os métodos de extração sugeriram até 8 (BIC e MAP) ou 11 fatores (Análise Paralela). Os modelos de 7, 8, 9, 10 e 11 fatores foram avaliados para o conjunto de 66 itens. 0 modelo de 9 fatores foi mais interpretável, entretanto em busca de itens com melhor qualidade, foi considerado um ponto de corte de 0,40 para as cargas fatoriais (Hair et al., 2009). Foram excluídos 19 itens com cargas fatoriais cruzadas.

Com os itens restantes, foi rodada uma nova análise fatorial exploratória. Assim, a escala passou a ter 47 itens, sendo que os métodos de extração sugeriram até 8 (BIC e MAP) ou 9 fatores (Análise Paralela). A solução com melhor interpretação teórica foi a de 7 fatores, as soluções de 8 e 9 fatores apresentaram poucos itens por fator, não sendo possível uma interpretação acurada dos dados. Na Tabela 1 estão apresentadas as cargas fatoriais, quantidade de itens e coeficiente alfa por fator da solução de 7 fatores. 0 modelo apresentou um bom ajuste com $\chi^{2}(1121)=5622.631(p<0,001)$, RMSEA $=$ $0,079$ (95\% IC: $0,077-0,081, p<0,001), \mathrm{CFI}=0,992$ e TLI $=0,989$. 
Tabela 1: Cargas Fatoriais, Quantidade de Itens e Coeficiente Alpha por Fator da Versão Adaptada

\begin{tabular}{|c|c|c|c|c|c|c|c|}
\hline Itens & Interpessoal & Carreira & $\begin{array}{l}\text { Falta de } \\
\text { suporte }\end{array}$ & $\begin{array}{l}\text { Desempenho } \\
\text { acadêmico }\end{array}$ & $\begin{array}{l}\text { Produção } \\
\text { científica }\end{array}$ & $\begin{array}{l}\text { Reconheci- } \\
\text { mento acadê- } \\
\text { mico }\end{array}$ & Instituição \\
\hline Item 13 & $0,595^{\star}$ & $-0,046$ & 0,006 & 0,033 & $0,116^{\star}$ & $0,272^{\star}$ & 0,020 \\
\hline Item 9 & $0,556^{\star}$ & $-0,049$ & 0,096 & 0,025 & $-0,023$ & $0,357 *$ & 0,032 \\
\hline Item 4 & $0,552^{*}$ & $0,098^{*}$ & $-0,183^{*}$ & 0,012 & $-0,045$ & $0,278^{*}$ & $-0,013$ \\
\hline Item 8 & $0,477^{*}$ & $-0,030$ & $-0,172^{\star}$ & $0,143^{*}$ & $0,206^{*}$ & 0,002 & 0,01 \\
\hline Item 21 & $0,130 *$ & $0,934^{*}$ & $0,081^{*}$ & $-0,134^{\star}$ & $-0,004$ & $-0,108^{*}$ & 0,019 \\
\hline Item 27 & $-0,006$ & $0,814^{*}$ & $-0,014$ & $-0,033$ & $-0,014$ & $0,112^{*}$ & 0,002 \\
\hline Item 15 & $0,131^{*}$ & $0,803^{*}$ & $0,145^{\star}$ & $-0,113^{*}$ & 0,047 & $-0,005$ & $-0,052$ \\
\hline Item 31 & 0,072 & $0,773^{*}$ & $-0,152^{\star}$ & $-0,038$ & $0,057^{*}$ & $0,114^{*}$ & $-0,011$ \\
\hline Item 37 & $-0,012$ & $0,602^{\star}$ & $-0,062$ & $0,117^{*}$ & 0,005 & $-0,063$ & $0,207^{*}$ \\
\hline Item 35 & $-0,113^{*}$ & $0,575^{\star}$ & 0,036 & $0,093^{*}$ & 0,024 & $0,291^{*}$ & 0,026 \\
\hline Item 24 & $-0,082^{\star}$ & $0,562^{*}$ & $0,244^{*}$ & $0,140^{\star}$ & $-0,046$ & $0,226^{*}$ & $-0,077^{\star}$ \\
\hline Item 10 & $0,141^{*}$ & $0,526^{*}$ & 0,016 & $-0,097 *$ & 0,037 & $0,152^{*}$ & $-0,021$ \\
\hline Item 26 & $-0,064$ & $0,469 *$ & $0,121^{*}$ & $0,143^{*}$ & 0,06 & $0,187 *$ & 0,008 \\
\hline Item 18 & $0,231^{*}$ & $0,124^{*}$ & $0,668^{*}$ & 0,057 & $-0,005$ & $0,103^{*}$ & 0,022 \\
\hline Item 12 & $0,347^{*}$ & 0,004 & $0,656^{*}$ & $-0,069$ & 0,062 & 0,032 & $-0,003$ \\
\hline Item 23 & 0,003 & 0,043 & $0,566^{\star}$ & $0,200 *$ & 0,067 & $-0,059$ & 0,015 \\
\hline Item 50 & 0,068 & 0,053 & $0,509 *$ & 0,031 & 0,062 & $0,119 *$ & $0,194^{\star}$ \\
\hline Item 20 & 0,08 & $0,299 *$ & $0,505^{\star}$ & $0,135^{\star}$ & $-0,038$ & $-0,160^{*}$ & 0,088 \\
\hline Item 19 & $-0,107$ & $-0,027$ & $0,505^{\star}$ & $0,264^{*}$ & $-0,105^{\star}$ & 0,016 & $0,166^{\star}$ \\
\hline Item 56 & $-0,087$ & $0,251^{*}$ & $0,433^{*}$ & 0,013 & $0,102^{*}$ & 0,127 & 0,086 \\
\hline Item 32 & $0,122^{*}$ & 0,012 & $-0,002$ & $0,895^{\star}$ & 0,036 & $-0,012$ & $-0,02$ \\
\hline Item 29 & $0,095^{\star}$ & $0,064^{*}$ & 0,009 & $0,876^{*}$ & $-0,012$ & $-0,019$ & $-0,026$ \\
\hline Item 47 & 0,034 & $-0,105^{\star}$ & $0,054^{\star}$ & $0,764^{\star}$ & $0,067^{*}$ & $0,072^{\star}$ & $0,180^{*}$ \\
\hline Item 14 & $0,141^{*}$ & $-0,008$ & $0,179 *$ & $0,711^{*}$ & 0,038 & 0,035 & $-0,007$ \\
\hline Item 7 & 0,008 & $-0,032$ & $0,224^{\star}$ & $0,705^{\star}$ & $-0,007$ & $-0,05$ & 0,015 \\
\hline Item 40 & $-0,04$ & $0,074^{\star}$ & $0,105^{\star}$ & $0,434^{\star}$ & $0,117^{*}$ & $0,195^{\star}$ & $0,203^{*}$ \\
\hline Item 64 & $-0,085^{\star}$ & $0,089 *$ & $-0,094^{\star}$ & $0,090 *$ & $0,946^{*}$ & $0,042^{*}$ & $-0,066^{*}$ \\
\hline Item 65 & $-0,075^{\star}$ & 0,019 & $-0,021$ & $0,095^{\star}$ & $0,945^{\star}$ & $-0,03$ & 0,003 \\
\hline Item 66 & 0,013 & $0,080 *$ & 0,016 & $-0,008$ & $0,924^{*}$ & $-0,026^{*}$ & 0,03 \\
\hline Item 63 & $-0,039 *$ & $0,057 *$ & $-0,041^{*}$ & 0,039 & $0,922^{*}$ & 0,031 & $-0,02$ \\
\hline Item 67 & $0,052^{*}$ & 0,027 & $0,079 *$ & $-0,066^{*}$ & $0,918^{*}$ & $-0,026$ & $0,082^{*}$ \\
\hline Item 61 & $0,125^{\star}$ & $-0,053^{\star}$ & 0,01 & $-0,025$ & $0,839 *$ & $0,045^{\star}$ & $0,133^{*}$ \\
\hline Item 62 & $0,150^{\star}$ & $-0,085^{\star}$ & $0,060^{*}$ & $0,042^{\star}$ & $0,801^{*}$ & 0,029 & $0,124^{\star}$ \\
\hline
\end{tabular}




\begin{tabular}{|c|c|c|c|c|c|c|c|}
\hline Item 60 & $0,157^{*}$ & 0,031 & 0,002 & 0,051 & $0,478^{*}$ & $0,369 *$ & $-0,006$ \\
\hline Item 54 & 0,034 & $0,142^{*}$ & $-0,088^{*}$ & $-0,098^{\star}$ & $0,139 *$ & $0,696^{*}$ & 0,119 \\
\hline Item 55 & $-0,025$ & 0,052 & $0,144^{*}$ & $-0,017$ & $0,098^{*}$ & $0,672^{\star}$ & 0,108 \\
\hline Item 53 & $0,177^{\star}$ & 0,029 & $-0,054$ & $-0,015$ & $0,105^{\star}$ & $0,637^{*}$ & $0,195^{\star}$ \\
\hline Item 57 & 0,016 & 0,01 & $0,291^{*}$ & $-0,046$ & $0,385^{\star}$ & $0,585^{\star}$ & $-0,170^{*}$ \\
\hline Item 59 & 0,009 & $-0,062^{*}$ & $0,289 *$ & 0,022 & $0,370 *$ & $0,577^{\star}$ & $-0,132^{\star}$ \\
\hline Item 39 & 0,035 & $-0,049$ & $0,093^{*}$ & $0,164^{*}$ & $-0,034$ & $0,491^{\star}$ & 0,326 * \\
\hline Item 52 & $-0,06$ & $0,249 *$ & 0,034 & $-0,027$ & $0,080 *$ & $0,483^{*}$ & $0,222^{*}$ \\
\hline Item 48 & $0,093^{*}$ & 0,018 & $-0,033$ & $0,166^{*}$ & $-0,051$ & $0,442^{\star}$ & $0,352^{*}$ \\
\hline Item 46 & 0,027 & $-0,072$ & 0,029 & $-0,052$ & $0,074^{*}$ & 0,014 & $0,915^{\star}$ \\
\hline Item 38 & $-0,038$ & 0,017 & 0,024 & 0,036 & $-0,043$ & $-0,021$ & $0,853^{*}$ \\
\hline Item 44 & $0,135^{\star}$ & $0,151^{*}$ & $0,090 *$ & $-0,008$ & $-0,011$ & $0,199 *$ & $0,470 *$ \\
\hline Item 51 & $-0,015$ & 0,005 & $0,166^{*}$ & 0,026 & $-0,041$ & $0,363^{*}$ & $0,454^{\star}$ \\
\hline Item 49 & 0,032 & $0,146^{*}$ & $-0,129 *$ & 0,090 * & 0,052 & $0,218^{*}$ & $0,415^{\star}$ \\
\hline $\begin{array}{l}\mathrm{N}^{\circ} \mathrm{de} \\
\text { itens }\end{array}$ & 4 & 9 & 7 & 6 & 8 & 8 & 5 \\
\hline$a$ & 0,61 & 0,90 & 0,82 & 0,91 & 0,97 & 0,90 & 0,82 \\
\hline
\end{tabular}

O primeiro fator envolveu motivos sobre a relação com colegas, assim foi denominado motivos interpessoais (Exemplo de item (13): "Não fazer amigos no programa/ universidade"). O segundo fator envolveu questões relacionadas à percepção da abertura do mercado de trabalho e salariais, assim foi denominado motivos relacionados à carreira (ex.:Item 21, "O mercado de trabalho ser muito limitado"). 0 terceiro fator avaliou questões relacionados à interação família, trabalho e formação acadêmica, sendo denominado motivos relacionados à falta de suporte (Item 18, "As exigências do curso e do meu trabalho serem incompatíveis"). 0 quarto fator está relacionado à questões de avaliações acadêmicas formais (e.g. notas em disciplinas), sendo denominado motivos relacionados ao desempenho acadêmico (Item 32, "Ter desempenho baixo em algumas disciplinas").

0 quinto fator englobou itens que avaliam dificuldade com a escrita científica, sendo denominado motivos relacionados à produção científica (Item 64, "Dificuldade em se focar na escrita"). 0 sexto fator abordou questões relacionados à percepção da pessoa como pesquisador e a da relevância de sua pesquisa, assim foi denominado de motivos relacionados ao reconhecimento acadêmico (Item 54, "Não ser reconhecido enquanto pesquisador"). 0 sétimo fator avaliou questões relacionadas à estrutura física e organizacional dos programas de pós-graduação, sendo denominado de motivos institucionais (Item 46, "Dificuldade de acesso à internet no campus").

\section{Análise dos itens pelo modelo de Rasch}

Os parâmetros das pessoas e dos itens pelo modelo Rasch estão apresentados na Tabela 2. Todos os fatores foram avaliados como sendo unidimensionais, segundo os critérios de Linacre (2014), em que a variância do primeiro contraste tem que ser menor que 2. Ainda foi verificado se havia funcionamento diferencial dos itens (DIF) entre grupos de bolsistas e não bolsistas, sendo encontrada apenas para o item 56 do Fator 3 ("Perder a bolsa"), sendo mais favorável para grupo de bolsistas $[t(367)=11,39, p<0,001]$. 
Tabela 2: Índices de Traço Latente, Ajuste dos Itens da Versão Adaptada

\begin{tabular}{|c|c|c|c|c|c|c|}
\hline Fator & Itens & Dificuldade (ઠ) & EP & Infit & Outfit & $r_{\text {item-theta }}$ \\
\hline \multirow{4}{*}{ F1 - Motivos interpessoais } & Item 13 & $-0,20$ & 0,05 & 0,94 & 0,91 & 0,75 \\
\hline & Item 9 & $-0,81$ & 0,05 & 0,82 & 0,83 & 0,80 \\
\hline & Item 4 & $-0,20$ & 0,05 & 0,99 & 1,02 & 0,72 \\
\hline & Item 8 & 1,13 & 0,07 & 0,82 & 0,83 & 0,80 \\
\hline \multirow{9}{*}{$\begin{array}{c}\text { F2 - Motivos relacionados à } \\
\text { carreira }\end{array}$} & Item 21 & $-0,40$ & 0,05 & 0,79 & 0,77 & 0,77 \\
\hline & Item 27 & $-0,24$ & 0,04 & 0,78 & 0,75 & 0,78 \\
\hline & Item 15 & $-0,49$ & 0,05 & 0,85 & 0,81 & 0,76 \\
\hline & Item 31 & 0,12 & 0,04 & 0,94 & 0,92 & 0,74 \\
\hline & Item 37 & 0,49 & 0,04 & 1,22 & 1,33 & 0,65 \\
\hline & Item 35 & 0,09 & 0,04 & 0,97 & 0,97 & 0,73 \\
\hline & Item 24 & $-0,16$ & 0,04 & 1,00 & 1,03 & 0,72 \\
\hline & Item 10 & 0,38 & 0,04 & 1,25 & 1,29 & 0,65 \\
\hline & Item 26 & 0,22 & 0,04 & 1,28 & 1,33 & 0,66 \\
\hline \multirow{7}{*}{$\begin{array}{l}\text { F3 - Motivos relacionados a } \\
\text { falta de suporte }\end{array}$} & Item 18 & 0,15 & 0,04 & 0,68 & 0,71 & 0,72 \\
\hline & Item 12 & 0,27 & 0,04 & 0,90 & 0,92 & 0,66 \\
\hline & Item 23 & $-0,22$ & 0,04 & 1,04 & 1,05 & 0,65 \\
\hline & Item 50 & 0,05 & 0,04 & 0,83 & 0,87 & 0,68 \\
\hline & Item 20 & 0,04 & 0,04 & 0,87 & 0,91 & 0,68 \\
\hline & Item 19 & 0,46 & 0,04 & 1,31 & 1,21 & 0,62 \\
\hline & Item 56 & $-0,74$ & 0,05 & 1,62 & 1,39 & 0,59 \\
\hline \multirow{6}{*}{$\begin{array}{l}\text { F4 - Motivos relacionados } \\
\text { ao desempenho acadêmico }\end{array}$} & Item 32 & 0,35 & 0,06 & 0,57 & 0,59 & 0,85 \\
\hline & Item 29 & 0,35 & 0,06 & 0,83 & 0,81 & 0,82 \\
\hline & Item 47 & 0,25 & 0,06 & 0,69 & 0,69 & 0,84 \\
\hline & Item 14 & $-0,23$ & 0,06 & 0,85 & 0.90 & 0.84 \\
\hline & Item 7 & $-0,51$ & 0,06 & 1,43 & 1,43 & 0,78 \\
\hline & Item 40 & $-0,20$ & 0,06 & 1,57 & 1,70 & 0,73 \\
\hline \multirow{8}{*}{$\begin{array}{l}\text { F5 - Motivos relacionados à } \\
\text { produção científica }\end{array}$} & Item 64 & $-0,33$ & 0,07 & 0,68 & 0,66 & 0,92 \\
\hline & Item 65 & 0,11 & 0,07 & 0,78 & 0,83 & 0,90 \\
\hline & Item 66 & $-0,17$ & 0,07 & 0,61 & 0,62 & 0,92 \\
\hline & Item 63 & $-0,23$ & 0,07 & 0,73 & 0,74 & 0,91 \\
\hline & Item 67 & $-0,08$ & 0,07 & 0,73 & 0,72 & 0,91 \\
\hline & Item 61 & 0,47 & 0,07 & 0,88 & 0,94 & 0,89 \\
\hline & Item 62 & 0,47 & 0,07 & 1,13 & 1,13 & 0,87 \\
\hline & Item 60 & $-0,23$ & 0,07 & 2,36 & 2,66 & 0,77 \\
\hline
\end{tabular}




\begin{tabular}{ccccccc}
\hline & Item 54 & $-0,24$ & 0,05 & 1,22 & 1,34 & 0,67 \\
& Item 55 & $-0,18$ & 0,05 & 0,79 & 0,81 & 0,77 \\
F6 - Motivos relacionados & Item 53 & 0,28 & 0,04 & 0,79 & 0,76 & 0,77 \\
ao reconhecimento acadê- & Item 57 & $-0,44$ & 0,05 & 1,02 & 1,01 & 0,73 \\
mico & Item 59 & $-0,35$ & 0,05 & 1,15 & 1,17 & 0,72 \\
& Item 39 & 0,25 & 0,04 & 1,17 & 1,36 & 0,69 \\
& Item 52 & 0,10 & 0,04 & 1,02 & 1,08 & 0,72 \\
& Item 48 & 0,60 & 0,04 & 1,22 & 1,34 & 0,67 \\
\hline \multirow{4}{*}{ F7 - Motivos institucionais } & Item 46 & 0,42 & 0,05 & 0,77 & 0,72 & 0,69 \\
& Item 38 & 0,47 & 0,05 & 1,10 & 1,04 & 0,69 \\
& Item 44 & $-0,34$ & 0,05 & 0,96 & 0,95 & 0,75 \\
& Item 51 & $-0,72$ & 0,05 & 1,01 & 1,04 & 0,76 \\
& Item 49 & 0,17 & 0,05 & 1,19 & 1,22 & 0,67 \\
\hline
\end{tabular}

Nota. EP = Erro Padrão; ritem-theta = correlação de cada item com o total da escala.

$\mathrm{Na}$ Tabela 2, é possível perceber que os itens 40 , 56 e 60 apresentaram índices de ajuste (Infit e Outfit) fora do esperado - 0,5 e 1,5 - estabelecido por Linacre (2014). De forma geral, os itens têm uma probabilidade de endosso média, sendo que o Item 9 do Fator 1 foi o mais fácil $(\delta=-0,81)$ e o Item 8 do Fator 1 o mais difícil $(\delta=1,13)$. Em relação à correlação-item total, o menor valor encontrado foi para o item 56, que apresentou um valor de 0,59. A consistência interna dos itens foi boa, com valores maiores que 0,90 . Em relação às pessoas, a consistência foi de moderada a alta, sendo menor no Fator $1(\mathrm{a}=0,54)$.

\section{DISCUSSÃO}

Esse manuscrito teve como objetivo adaptar a escala de motivos para evasão no ensino superior (E-MES) para a Pós-Graduação Strictu Senso (M-PG). Os resultados encontrados indicaram que a M-PG apresentou propriedades psicométricas adequadas e permite a avaliação dos motivos de evasão para o contexto em que foi adaptada. A análise da estrutura interna sugeriu entre oito e 11 grupos de fatores/causas de provável abandono do curso de pós-graduação. Dentre os modelos testados, o de 7 fatores foi o mais interpretável

Os principais motivos agrupados na análise fatorial estão relacionados com questões interpessoais, carreira, falta de suporte, desempenho acadêmico, produção científica, reconhecimento acadêmico e motivos institucionais. Esses fatores correspondem ao que Tinto $(1975 ; 2007)$ afirmava sobre o desempenho acadêmico e integração ao ambiente, que engloba questões de relacionamentos interpessoais. Complementar a isso, estão os motivos sobre enriquecimento curricular e a falta de suporte (Demetriou \& Schmitz-Sciborski, 2011) e desempenho acadêmico e pressão por produtividade (Leijen et al., 2015).

A análise de Rasch (Bond \& Fox, 2015) permitiu a identificação da unidimensionalidade dos fatores e a ausência de DIF nos itens, com exceção do item 56. 0 funcionamento diferencial desse item é compreensível em virtude do conteúdo do mesmo. Afinal, ser aluno bolsista, implica em ter dedicação exclusiva nas atividades dos PPGs e não permite ao aluno ter vínculo empregatício com outras instituições ou empresas (Coordenação de Aperfeiçoamento de Pessoal de Nível Superior [CAPES], 2012) o que faz da bolsa de estudos a única renda desse pós-graduando.

No que diz respeito ao ajuste dos itens ao modelo, três deles $(40,56$ e 60) apresentaram valores fora do esperado (Linacre, 2014). Os índices de ajustes mais empregados no modelo são o infit e outfit. 0 primeiro refere-se a discordâncias em relação ao traço latente, indicando que uma maior quantidade inesperada de erros e acertos implica no aumento desse índice. Por sua vez, o outfit diz respeito a diferenças inesperadas entre a dificuldade do item em relação ao logit de habilidade do indivíduo, ou 
seja, pessoas com um nível baixo de habilidade endossam itens de alta dificuldade, ou vice-versa (Nakano et al., 2015). Contudo, uma análise qualitativa dos itens permite a compreensão e justificam a sua manutenção. 0 item 40 investiga sobre a dificuldade de compreender conteúdos de trabalhos na pós-graduação e o item 60 é sobre sentimento de inferioridade em relação aos outros alunos do programa. É esperado que as pessoas endossem esses itens pois são conteúdos relacionados a motivos muito específicos com a crença na própria capacidade e que não exigem altos níveis de habilidade. Caso as situações ocorram, as pessoas tendem a endossá-los, pois apesar de desejarem uma carreira bem-sucedida, sentir-se competente e ao mesmo nível dos seus pares aparece como algo essencial para a realização da pósgraduação.

No que diz respeito à comparação com a escala original (Ambiel, 2017), cinco fatores permaneceram inalterados quanto ao conteúdo e aos itens que os compunham, e dois foram alterados quanto a estes aspectos. Os fatores motivos vocacionais e relacionados à autonomia foram substituídos pelos fatores motivos relacionados à produção científica e relacionados ao reconhecimento acadêmico. Esses novos fatores foram compostos pelos itens construídos baseados nos depoimentos de alunos de pós-graduação e nas sugestões dos juízes. Além disso a quantidade de itens alterou, a escala original possui 53 itens e a adaptada 47. Essas alterações são compreensíveis pensando-se na especificidade do contexto de Pós-graduação (Castelló et al., 2017).

A carreira científica é importante para o desenvolvimento tecnológico de qualquer país, sendo necessário garantir que pessoas capazes e envolvidas com a pesquisa concluam seus cursos de pós-graduação a fim de contribuírem para esse crescimento. Os entraves que dificultam esse processo são diversos e envolvem questões de relacionamentos interpessoais, estrutura das instituições e oportunidades de desenvolvimento de pesquisas. Isso causa prejuízos emocionais para os pós-graduandos, além de financeiro para instituições de ensino e financiadoras de pesquisas. Este artigo se propôs a compreender quais os principais fatores que contribuem para a evasão na pós-graduação. 0 que se sobressaiu nesta investigação foi a importância de esses estudantes sentirem segurança na própria carreira, tanto em termos financeiros como na capacidade de desenvolverem atividades de pesquisa e serem reconhecidos como pesquisadores, o que foi refletido na estrutura fatorial da escala.
É importante salientar que este estudo não pretende esgotar o assunto e sim instigar a investigação das causas de abandono de cursos de pós-graduação. Ainda que traga informações importantes, algumas lacunas devem ser destacadas para que possam ser investigadas futuramente. Dentre elas, estão a baixa representatividade amostral de alunos que abandonaram seus cursos. Além disso, não foram verificados os motivos com base nas notas avaliativas da CAPES, pois entende-se que programas com maiores notas exigem mais dos seus alunos. Entretanto, tais limitações não diminuem a contribuição desse instrumento para auxiliar profissionais a pensarem em intervenções no contexto da pós-graduação, no sentido de melhorar a percepção subjetiva e desenvolvimento acadêmico dos estudantes.

Considerando futuras pesquisas acerca do assunto, é importante mencionar a relação desses motivos com outros fatores, como satisfação com a vida e com o trabalho e quais tipos de intervenções podem ser mais eficazes para minimizar a evasão de futuros pesquisadores na pós-graduação. Entende-se que tais lacunas são investigações que demandam tempo e colaboração de outros atores sociais, como coordenações, docentes e até mesmo os próprios órgãos de fomento que podem verificar, de forma mais específica, e intervir de maneira direta na evasão na pós-graduação, investindo em melhorias e evitando assim um déficit aos cofres públicos.

\section{DECLARAÇÃO DA CONTRIBUIÇÃO DOS AUTORES}

Os cinco autores contribuíram com a análise formal dos dados, conceitualização, investigação e metodologia. A.R.LC. foi responsável pelo gerenciamento da pesquisa. S.R.Z foi responsável pela revisão.

\section{DECLARAÇÃO DE CONFLITOS DE INTERESSE}

Declaramos que não há conflito de interesse envolvido no desenvolvimento da pesquisa.

\section{REFERÊNCIAS}

Almeida, E. C. E. \& Guimarães, J. A. (2013). Brazil's growing production of scientific articles: How are we doing with review articles and other qualitative indicators? Scientometrics, 97(2), 287-315. https:// doi.org/10.1007/s11192-013-0967-y 
Ambiel, R. A. M. (2015). Construção da escala de motivos para evasão no ensino superior. Avaliação Psicológica, $14(1), 41-52$.

Ambiel, R. A. M. (2017). Escala de motivos de Evasão do Ensino Superior (M-ES). Editora Hogefre.

Asún, R. A., Rdz-Navarro, K., \& Alvarado, J. M. (2016). Developing multidimensional Likert scales using item factor analysis: The case of four-point items. Sociological Methods \& Research, 45(1), 109-133. https://doi.org/10.1177/0049124114566716

Bond, T. G., \& Fox, C. M. (2015). Applying the Rasch model fundamental measurement in the human sciences (3rd ed.). Routledge.

Castelló, M., Pardo, M., Sala-Bubaré, A., \& Suñe-Soler, N. (2017). Why do students consider dropping out of doctoral degrees? Institutional and personal factors. Higher Education, 74, 1053-1068. https://doi. org/10.1007/s10734-016-0106-9

Coordenação de Aperfeiçoamento de Pessoal de Nível Superior (CAPES). (2012). Portaria No 181, de 18 de Dezembro de 2012 - Regulamento do Programa de Suporte à Pós Graduação de instituições de Ensino Particulares. https://www.capes.gov.br/images/ stories/download/legislacao/Portaria_181_de18122012.pdf

Damásio, B. F. (2012). Uso da análise fatorial exploratória em psicologia. Avaliaçao Psicologica: Interamerican Journal of Psychological Assessment, 11(2), 213-228.

Demetriou, C., \& Schmitz-Sciborski, A. (2011). Integration, motivation, strengths and optimism: Retention theories past, present and future. Em R. Hayes (Org.), Proceedings of the 7th National Symposium on student retention (pp. 300-312). University of Oklahoma, Norman, OK, United States. https://studentsuccess. unc.edu/student-success-literature/demetriou-andschmitz-sciborski-2/

Duque, J. C., Brondani, J. T., \& Luna, S. P. L. (2005). Estresse e pós-graduação em Medicina Veterinária. Revista Brasileira de Pós-Graduação, 2(3), 134-148. https://doi.org/10.21713/2358-2332.2005.v2.63
Faro, A. (2013). Estresse e estressores na pós-graduação: estudo com mestrandos e doutorandos no Brasil. Psicologia: Teoria E Pesquisa, 29(1), 51-60. https:// doi.org/10.1590/S0102-37722013000100007

Ferreira, J. S., Oliveira, L. A., Godinho, R. L. P., Santos, P. S. S. R., Hanzelmann, R. S., \& Passos, J. P. (2016). Alunos da pós-graduação em enfermagem e o nível de estresse. Pró-UniverSUS, 7(3), 20-25.

Gardner, S. K. (2009). Student and faculty attributions of attrition in high and low-completing doctoral programs in the United States. Higher Education, 58(1), 97-112. https://doi.org/10.1007/s10734-008-9184-7

GEOCAPES - Sistema de Informações Georreferenciadas, Coordenação de Aperfeiçoamento de Pessoal de Nível Superior (CAPES). (2018). https://geocapes. capes.gov.br/geocapes/\#

Hair, J. F., Anderson, R. E., Tatham, R. L., \& Black, W. C. (2009). Análise multivariada de dados. Bookman.

Lai, K., \& Green, S. B. (2016). The problem with having two watches: Assessment of fit When RMSEA and CFI disagree. Multivariate Behavioral Research, 51, 220239. https://doi.org/10.1080/00273171.2015.11343 06

Leijen, Ä., Lepp, L., \& Remmik, M. (2015). Why did I drop out? Former students' recollections about their study process and factors related to leaving the doctoral studies.StudiesinContinuingEducation,38(2),129-144. https://doi.org/10.1080/0158037X.2015.1055463

Levecque, K., Anseel, F., De Beuckelaer, A., Van der Heyden, J., \& Gisle, L. (2017). Work organization and mental health problems in PhD students. Research Policy, 46(4), 868-879. https://doi.org/10.1016/j. respol.2017.02.008

Linacre, J. M. (2014). Winsteps Rash measurement computer program. http://www.winsteps.com/index. $\mathrm{htm}$

Malagris, L. E. N., Suassuna, A. T. R., Bezerra, D. V., Hirata, H. P., Monteiro, J. L. F., Silva, L. R., Lopes, M. C. M., \& Santos, T. S. (2009). Níveis de estresse e características sociobiográficas de alunos de pósgraduação. Psicologia em Revista, 15(2), 184-203. https://doi.org/10.5752/P.1678-9563.2009v15n2p184 
Nakano, T. C., Primi, R., \& Nunes, C. H. S. S. (2015). Análise de itens e Teoria de Resposta ao Item (TRI). Em C. S. Hutz, D. R. Bandeira, \& C. M. Trentini (Orgs.), Psicometria (pp. 97-124). Artmed.

Paulino, C. A., Prezotto, A. O., Frias, A. C., Bataglia, P. R., \& Aprile, M. R. (2010). Sintomas de estresse e tontura em estudantes de pós-graduação. Revista Equilíbrio Corporal E Saúde, 2(1), 15-26.

Stubb, J., Pyhältö, K., \& Lonka, K. (2011). Balancing between inspiration and exhaustion: PhD students' experienced socio-psychological well-being. Studies in Continuing Education, 33(1), 33-50. https://doi. org/10.1080/0158037X.2010.515572

Tinto, V. (1975). Dropout from higher education: A theoretical synthesis of recent research. Review of Educational Research, 45(1), 89-125. https://doi. org/10.3102/00346543045001089
Tinto, V. (2007). Research and practice of student retention: what next? Journal of College Student Retention, 8(1), 1-19. http://citeseerx.ist.psu.edu/viewdoc/ download?doi=10.1.1.133.2661\&rep=rep1\&type=pdf

Van der Haert, M., Ortiz, E. A., Emplit, P., Halloin, V., \& Dehon, C. (2014). Are dropout and degree completion in doctoral study significantly dependent on type of financial support and field of research? Studies in Higher Education, 39(10), 1885-1909. https://doi.org $/ 10.1080 / 03075079.2013 .806458$

Data de submissão: 10.10 .2018 Data da primeira decisão editorial: 17.05.2019 Aprovado em: 24.06.2019 